\title{
The Current Situation and Development Trend of Micro-/nano-sensor and MNPTS
}

\author{
Jinyong Zhu, Lidong Wang \\ Department of Electronic and Optical Engineering, Mechanical Engineering \\ College, 050003, Shijiazhuang Hebei \\ 237706847@qq.com
}

\section{Keywords: Micro-/nano-sensor, MNPTS, MEMS}

\begin{abstract}
In recent years, with the development of microelectronics technology of mechanical system, micro-/nano-sensor based on micro electro mechanical system (MEMS) technology has been widely used in the field of national defense and civil. Micro-/nano-sensor, especially in the micro air vehicles (MAVs) navigation timing and positioning system has become one of the important direction of development at home and abroad. In this paper, the relationship between MNPTS and MAVs is introduced, the research situation and development trend of micro-/nano-sensor and MNPTS are summarized.
\end{abstract}

\section{Introduction}

In the modern war, MAVs in the low altitude areas (within the atmosphere) is used more and more widely, as a key development direction of the air combat weapon and the ground detection, surveillance and attack weapon. And it is very likely that use of MAVs swarm to attack or defense combat mode. Among them, the navigation system is one of the core information source of the MAVs, which is an important guarantee for the realization of the single navigation, group of navigation and cooperative engagement, and achieve its reconnaissance, surveillance and attack targets. Therefore, about the MAVs navigation technology research, the emphasis is on research of small volume, light weight, high precision, suitable for the MAVs autonomous navigation system based on micro-/nano-sensor(MEMS gyro and accelerometer, nano photonic gyro/photonic crystals gyro, photonic crystal fiber optic gyro(FOG), atomic gyroscope and accelerometer, etc) of the miniature navigation positioning timing system (MNPTS), which have important practical and long-term significance.

\section{MNPTS overview}

MNPTS will meet the requirements of the MAVs in terms of volume, weight and power consumption, etc

The MAVs has the characteristics of small volume, light weight (generally no more than one-two $\mathrm{kg}$ ), low power consumption, etc. In order to increase the battery life, improve its combat capability, etc, navigation system must meet the requirements of smaller volume, lighter weight and lower power consumption, and based on micro-/nano-sensor MNPTS just have these characteristics. For example, AD company, the MEMS inertial measurement unit ADIS16375 has three gyroscope and accelerometer, gyroscope and accelerometer zero bias stability of $12 \% \mathrm{~h}, 0.1 \mathrm{mg}$ respectively, and the size is $44 \mathrm{~mm} * 14 \mathrm{~mm} * 47 \mathrm{~mm}$; At the university of Michigan recently developed the timing of inertial measurement unit(TIMU) integrated in the narrow space of the $10 \mathrm{~mm} 3$, power consumption is less than 1w. And with the development of micro-/nano-sensor technology, MNPTS weight will be lighter, volume will be smaller, power consumption will be lower, can fully meet the requirements of the MAVs.

MNPTS can realize the MAVs autonomous navigation and synergy with high precision attitude, navigation, positioning and timing information,

At present, the micro-/nano-sensor is still in the tactical level, and the drift error of MNPTS is large, also does not have long time navigation ability. However, this kind of situation is changing 
fast. Such as the United States DARPA launched "the navigation and positioning and timing Micro technology(Micro-PNT)",which is about miniaturization and high precision clock and inertial navigation technology research project, this project aims to establish volume less than $1 \mathrm{~cm} 3$, power consumption less than $30 \mathrm{mw}$,frequency accuracy of $1 \times 10-15$, stability of $1 \mathrm{~ns} /$ day of atomic frequency reference unit, and the total volume is less than $1 \mathrm{~cm} 3$, power consumption is less than $5 \mathrm{mw}$, angle random walk (ARW) is less than $0.001^{\circ} / \mathrm{h} 0.5$, offset is less than $0.01^{\circ} / \mathrm{h}$ navigation level integrated Micro gyroscope, CEP is about $1.852 \mathrm{~km} / \mathrm{h}$ single chip independent navigation devices. From individual to all kinds of combat platforms, from missile, precision-guided munitions to all kinds of unmanned aerial vehicles, unmanned submersible to provide GPS services when limited under the condition of accurate positioning and navigation services, especially for high dynamic (not less than $1000 \mathrm{~km} / \mathrm{h}$ ) under the condition of short supply accurate positioning and navigation service. At the same time, it is helpful to realize the MAVs group synergy as the azimuth and elevation benchmarks.

\section{MNPTS also can satisfy the requirement of attitude control and precision with providing high} accuracy navigation information,

Micro-/nano-sensor and MNPTS, can provide the missile, precision-guided munitions, the different kinds of attitude control and precision-guided unmanned aerial vehicles for high precision attitude and heading position and other navigation information, to ensure its implementation tactical objectives and complete its task.

\section{The current situation and trend development of foreign micro-/nano-sensor and MNPTS}

The rapid development of the western developed countries in the field of micro-/nano-sensor and MNPTS. At present, the technical performance index is gradually from the tactical level to the navigation level. Especially in the United States, the United States department of defense advanced research projects agency (DARPA) launched in January 2010, called "the navigation and positioning and timing Micro technology (Micro-PNT)", which is about miniaturization and high precision clock and inertial navigation technology research project, namely Micro PNT plan. This plan develops independent chip level inertial navigation and precision guidance technology and devices without relying on GPS. Intended for from individual to a variety of combat platforms, from missiles and precision-guided munitions to all sorts of unmanned aerial vehicles, unmanned submersible provide GPS services when limited under the condition of accurate positioning and navigation services, especially for high dynamic (not less than $1000 \mathrm{~km} / \mathrm{h}$ ) under the condition of short supply accurate positioning and navigation service. With a focus on the development of autonomous chip level of inertial navigation and precision guidance technology, micro calibration technology, etc. The goal is to develop volume less than $8 \mathrm{~mm} 3$, power consumption is less than $1 \mathrm{w}$ timing and inertial measurement unit, including size, weight, power consumption and cost is the key index. The project research mainly includes the following three aspects:

(1) The study of atomic clock with ultra small, low power consumption and high stability and accuracy

In 2002, DARPA started funding chip level atomic clocks (CSAC) technology research and development. The technical indicators are: volume is less than $1 \mathrm{~cm} 3$, power consumption is less than $30 \mathrm{mw}$, the precision is $1 \times 10-11$, stability of $1 \mathrm{~ns} /$ day. For weapons, weapon system and navigation system provides complete atomic clocks. In 2008 started funding integrated micro main atomic clocks technology (IMPACT) of research work, two orders of magnitude more CSAC performance .

(2) Inertial sensor and system research

Three research work mainly includes: one is the study of navigation level integrated micro gyroscope (NGIMG), which aims to research and development of miniature, low power consumption speed sensor, it can achieve long-term (hours to several days) and high precision navigation. Target (not chip level) : total volume is less than $1 \mathrm{~cm} 3$ (excluding power), power consumption is less than $5 \mathrm{mw}$, angle random walk is less than $0.001 \% / \mathrm{h} 0.5$, offset is less than $0.01 \%$, etc; Second, the research of micro inertial navigation technology, research and 
development can be directly measured inertia among variables, such as speed and distance sensors, high precision navigation system to alleviate the integral separate accelerometer and gyroscope signal to increase the error of the problems. Including the volume of the speed sensor is less than $1 \mathrm{~cm} 3$, power consumption is less than $5 \mathrm{mw}, 36$ hours on foot after positioning accuracy is $1 \mathrm{~m}$, each step speed induction deviation for $10 \mu \mathrm{m} / \mathrm{s}$. The third is study of tethered information micro automatic rotating platform (IT-MARS), which is used for debugging and demonstration of micro electro mechanical system (MEMS).

(3) Study of the combination of chip level atom navigator

In April 2012, DARPA issued "the combination of chip level atom navigator (C-SCAN)" subproject tender, seeking to inertial navigation sensor with different physical coordination, integrating into a single micro inertial measurement unit technology solutions and the miniaturization of atomic inertia benchmark, combination algorithm and the architecture and the combination of chip level atom navigator micro system integration and validation for three technology breakthroughs. The combination of gyro technical indexes for: in the range of $40 \mathrm{~Hz}$ (or about $15000 \% \mathrm{~s})$ and $1000 \mathrm{~g}$, within the scope of the bias stability of $10-4(\% / \mathrm{h})$ to $10-6(\% / \mathrm{h})$, the angle random walk $5 \times 10-4(\circ / \mathrm{h} 0.5)$, and the random walk is $5 \times 10-4(\mathrm{~m} / \mathrm{s} / \mathrm{h} 0.5)$, bias and scale factor drift of one over one million respectively.

At present, the micro inertial measurement unit in the performance of the MEMS accelerometer has reached the medium accuracy, and the bias stability is $2 \sim 3 \mu \mathrm{g}$. such as the Swiss Colibrys company, publicly reported high-performance inertial navigation level of micromechanical accelerometer the first time in 2012: plus or minus 15g range within the scope of the impact of up to $4000 \mathrm{~g}$, the bias stability of $10 \mu \mathrm{g}$, bias temperature coefficient is $0.2 \mathrm{mg} /{ }^{\circ} \mathrm{C}$, the temperature coefficient of the scale factor is $100 \mathrm{ppm} /{ }^{\circ} \mathrm{C}$, noise equivalent acceleration is less than $2 \mu \mathrm{g} / \mathrm{Hz} 1 / 2$, which is fully meet the demand of application in the field of inertial navigation. The United States of MEMS accelerometer products in precision can meet the application requirements of strategic missiles. Such as micro mechanical accelerometer on the project to achieve the accuracy of $1 \times 10-4 \mathrm{~g}$, the potential precision can also improve an order of magnitude; In recent years, the MOEMS (Micro -optical electronic mechanical system) accelerometer has become research focus in the field of inertial sensor. In 2008, the United States reported the Sandia national laboratories has a micron grating detection mechanical accelerometer, the noise equivalent acceleration at $1 \mathrm{kHz}$ to $24.4 \mu \mathrm{g} / \mathrm{Hzl} / 2$; Atomic accelerometer based on cold atom interferometer with its incomparable precision performance has become another hotspot; in the near future, NEMS (nano-electrical mechanical system) accelerometer with its smaller size and lower power consumption will gradually replace the MEMS accelerometer.

On December 18, 2010, the United States Atlantic inertial systems company successfully developed MinIM micro inertial measurement unit. Its diameter and length are $28 \mathrm{~mm}$, which has been used in the inertial guidance of small ammunition; In 2011, the British Goodrich Co has launched a new micro navigation device of $\mu \mathrm{Nav}$, which is integrated with MinIM micro inertial device and GPS chip, that is a high-performance GPS/INS combined navigation device, the size of a quarter of the latest production of similar units, has been widely applied to weapons guidance. It is an important milestone in the development of weapon guidance system.

\section{The current situation and trend development of domestic Micro-/nano-sensor and MNPTS}

\section{The domestic present situation and the development trend of Micro-/nano-sensor}

From "Nine Five", the domestic has been carrying out a research into the micro inertial sensors. The research unit is more, but there is a certain gap in technical performance indicators and the application level with the western developed countries. Among them, the technical performance index of the MEMS gyroscope and accelerometer is not high, still in the initial stage. Developed by Tsinghua university has began to develop micro silicon accelerometer. In 2012, the Beijing university of aeronautics and astronautics proposed a new micro mechanical accelerometer based on planar nano gratings; other micro-/nano-sensor are in the laboratory and research stage [1-4]. 


\section{The domestic present situation and the development trend of MNPTS}

MEMS inertial system is divided into inertial measurement unit (IMU) and inertial navigation system (INS). Using MEMS-IMU and GPS to constitute the SINS/GPS integrated navigation system, it is the main form of MEMS-INS application. This is due to: on the one hand, the MEMS-IMU precision is low, its composition of SINS can only maintain valuable seconds of navigation data, integrated navigation technology is the most cost-effective way to improve the performance of MEMS-INS; on the other hand, the MEMS-IMU greatly reduce the cost of the combined system of SINS, the MEMS integrated navigation system with cost advantages, promote the development and application of the integrated navigation technology. In recent years, the performance of tactical level MEMS-IMU is further improved, and the volume, weight and cost are gradually reduced.

At present, in the domestic research and development and production on the basis of MEMS gyroscope and MEMS accelerometer, already qualified MEMS - IMU innovative marketing ability of research and development and small batch production. However, due to the difference between MEMS and IC, the IMU-MEMS and SINS/GPS-MEMS integrated navigation system is difficult to be processed in the same chip, that is, the SINS/GPS-MEMS integrated navigation system is still in the research stage. Tsinghua university make use of U.S. BEI company's micro resonator gyro and IC silicon micro accelerometer Sensors developed MEMS-IMU, on-board experiments show that the positioning error of combination system $(\mathrm{CEP}<4 \mathrm{~m})$ is better than that of GPS positioning error $(\mathrm{CEP}=15 \mathrm{~m})[5]$, and the combined system can maintain high positioning accuracy $(\mathrm{CEP}=1.61 \mathrm{~m})$ when the GPS is short .Shanghai Jiao tong university using 3DM-GX1 MEMS device and OEMV-1G type receiver loose combination system is developed, on-board experiments show that the combined system in satellite is in good condition when the positioning accuracy is better than that of $2 \mathrm{~m}$; the position error of MEMS-IMU independently for $150-200 \mathrm{~m}$ when satellite failure $30 \mathrm{~s}$; Harbin engineering university of MEMSAHRS/GPS combination tight fusion filtering technology is studied.

\section{Summary}

Micro-/nano-sensor and MNPTS is one of the leading edge of the mainstream technology at home and abroad, the study of micro-/nano-sensor miniaturization, high precision to promote the development of the miniature intelligent vehicle. Analysis of the status of domestic and foreign research technology, hoping to provide reference for researchers in relevant field.

\section{References}

[1] Zhengping Zhao, New Development of the Typical MEMS and Wearable Sensor Technical, Micronanoelectronic Technology. 1 (2015)1-13.

[2] Huilan Liu, Yong Wng, Yichuang Tang, Lishuang Feng.Novel adjustment method for micro-mirrors in MOEMS gyroscope spatial resonator, Journal of Chinese Inertial Technology.4(2013)549-552.

[3] Heng Lin, Huilan Liu, Dewei Yang, Chunxi Zhang, Technologies and Development of MOEMS Inertial Sensors, Journal of Chinese Inertial Technology.2(2005)85-90

[4] Bo Fu, Yueyue Zhao, Overview About Recent Development Of MOEMS, Sensor World.(2004)

[5] Shifeng HU, Jiancang MA, Development of Micro Inertial Measurement Unit Based on MEMS Gyroscopes and Accelerometer, Aeronautical Computing Technique.6(2008) 115-119

[6] Hongguang LI, Progress on technologies of MEMS inertial sensors for modern military, Transducer and Microsystem Technologies.8(2014)5-19

[7] Juan Zhang, High-precision Micro-optical Accelerometer Signal Processing System, Master Dissertation of ZheJiang University.(2013) 
[8] Bo Li, Yongjun Yang, Yongqing Xu, Shujing Xu, Chao Xu, Hongtao He, Rong Luo, Xinyan Lu, An Integrated Silicon MEMS Vibration Gyroscope, Micronanoelectronic Technology. 2013

[9] Barbour N, Hopkins R, Kourepenis A, Inertial MEMS Systems and Applications. NATO Lecture series, RTOEN-SET-116, Low-Cost Navigation Sensors and Integration Technology, 2011.

[10] Tracy D Hudson, Deanna K.MEMS component research and development for army missile application,Proc of SPIE 1999.

[11] Liu J, Li J. Application technology of MEMS inertial system[C]. Proceeding of intelligent system design and engineering application of intelligent system design and engineering application. Changsha, China, 2010. 